\title{
Computational and traveling wave analysis of Tzitzéica and Dodd-Bullough-Mikhailov equations: An exact and analytical study
}

https://doi.org/10.1515/nleng-2021-0021

Received Apr 11, 2021; accepted Jul 22, 2021.

\begin{abstract}
Computational and travelling wave solutions provide significant improvements in accuracy and characterize novelty of imposed techniques. In this context, computational and travelling wave solutions have been traced out for Tzitzéica and Dodd-Bullough-Mikhailov equations by means of $\left(1 / G^{\prime}\right)$-expansion method. The different types of solutions have constructed for Tzitzéica and DoddBullough-Mikhailov equations in hyperbolic form. Moreover, solution function of Tzitzéica and Dodd-BulloughMikhailov equations has been derived in the format of logarithmic nature. Since both equations contain exponential terms so the solutions produced are expected to be in logarithmic form. Traveling wave solutions are presented in different formats from the solutions introduced in the literature. The reliability, effectiveness and applicability of the $\left(1 / G^{\prime}\right)$-expansion method produced hyperbolic type solutions. For the sake of physical significance, contour graphs, two dimensional and three dimensional graphs have been depicted for stationary wave. Such graphical illustration has been contrasted for stationary wave verses traveling wave solutions. Our graphical comparative analysis suggests that imposed method is reliable and powerful method for obtaining exact solutions of nonlinear evolution equations.
\end{abstract}

Keywords: Tzitzéica equation, $\left(1 / G^{\prime}\right)$-expansion method, Dodd-Bullough-Mikhailov equation, traveling wave solutions, exact solutions

\footnotetext{
Hülya Durur, Department of Computer Engineering, Faculty of Engineering, Ardahan University, Ardahan, 75000, Turkey Asıf Yokuş, Department of Mathematics, Faculty of Science, Firat University, Elazig, 23200, Turkey

*Corresponding Author: Kashif Ali Abro, Department of Basic Sciences and Related Studies, Mehran University of Engineering and Technology, Jamshoro, Pakistan, E-mail: kashif.abro@faculty.muet.edu.pk
}

\section{Introduction}

Nonlinear evolution equations are studied in many areas such as physics, engineering, social sciences and chemistry. Particularly in the last years, many studies have been conducted to attain either exact or analytical solutions of nonlinear evolution equations. Analytic solutions of nonlinear evolution equations have been investigated and traced out by means of several mathematical methods. For the sake of confirmation and evaluations, Baskonus and Cattani [1, 2] invoked extended sinh-gordon equation expansion method. Improved Bernoulli sub-equation function method has been employed by Bulut and Dusunceli [3, 4] via fractional and non-fractional appoaches. Maih et al. [5] suggested $\left(G^{\prime} / G-1 / G\right)$-expansion method for closed form traveling wave solutions of integro-differential equations. Yokus and Durur presented (1+1)-dimensional resonant nonlinear Schrödinger's equation by means of $\left(G^{\prime} / G\right)$ expansion method in [6-8]. Same author worked on sub equation method in [9] and $\left(1 / G^{\prime}\right)$-expansion method in [10-12]. The partial solutions via decomposition method and residual power series method have been found for solitary and periodic solutions for the coupled higherdimensional Burgers equations in [13-17]. Additionally Sumudu transform method is employed in [18]. The numerical study and three-dimensional elliptic partial differential equations have been haar wavelet collocation method [19, 20] and first integral method [21]. The most important advantage of the expansion methods is the generation of traveling wave solutions of partial differential equations, such solutions are difficult for investigation. The most important common feature of the expansion methods are easy to apply and useful for traditional wave transform. With the help of this transformation, partial differential equation can be converted into an ordinary differential equation. In this continuity, we consider the Tzitzéica equation of the form [22]:

$$
u_{t t}-u_{x x}-e^{u}+e^{-2 u}=0
$$


We consider Dodd-Bullough-Mikhailov equation [22]

$$
u_{x t}+e^{u}+e^{-2 u}=0 \text {. }
$$

Equations (1-2) play an important role in the field of solidstate physics and nonlinear optics. Various studies on equations (1-2) have been carried out by many researchers; for instance, Kumar et al. [23] have explored traveling wave solutions of the Tzitzéica type equations by invoking sine-Gordon expansion method. The new types of exact solutions and class of solitary-like solutions of the Tzitzéica equation have been explored by means of similarity reduction and classical Lie symmetry analysis by $\mathrm{Hu}-$ ber [24]. Solitons and periodic solutions have been obtained for equations (1-2) using tan $\mathrm{h}$ method in [25] in which analytic solutions for equations (1-2) are obtained using improved $\tan (\phi(\xi) / 2)$. Moreover, recent attempts have been explored on the basis of variety of the solutions as expansion method [26], exponential rational function method [27], $\exp _{a}$ function method [28], dressing factor method [29]. In brevity, we derived and obtained analytic solutions of equations (1-2) using (1/G')-expansion method. Additionally, the recent attempts of analytical techniques and exact solutions can be traced out [3032, 34-43] and [44-47] therein.

\section{$2\left(1 / G^{\prime}\right)$-Expansion method}

We get the general form of non-linear partial differential equation

$$
\sigma\left(u, \frac{\partial u}{\partial t}, \frac{\partial u}{\partial x}, \frac{\partial^{2} u}{\partial x^{2}}, \ldots\right)=0,
$$

in the general form. Here, let $u=u(x, t)=U(\xi), \quad \xi=$ $x+v t, \quad v \neq 0$, where $v$ is a constant and the velocity of the wave. One can convert into following nonlinear ordinary differential equation (ODE) for $U(\xi)$ :

$$
\tau\left(U, U^{\prime}, U^{\prime \prime}, U^{\prime \prime \prime}, \ldots\right)=0 .
$$

The solution of Eq. (4) is assumed to have the form

$$
U(\xi)=a_{0}+\sum_{i=1}^{N} a_{i}\left(\frac{1}{G^{\prime}}\right)^{i},
$$

where $a_{i}, \quad(i=0,1, \ldots, N)$ are constants and $G=G(\xi)$ provides the following second-order integer order differential equation as

$$
G^{\prime \prime}+\lambda G^{\prime}+\mu=0,
$$

where $\lambda$ and $\mu$ are constants to be determined after,

$$
\frac{1}{G^{\prime}(\xi)}=\frac{1}{-\frac{\mu}{\lambda}+A \cosh [\xi \lambda]-A \sinh [\xi \lambda]},
$$

where, $A$ is integral constant. Some constraints are needed for the solution of Eq. (7). These restrictions are the ignoring of values that makes the solution undefined. The asymptotic behavior of the solution to include a singular point is attractive for employees. In addition, the existence of a single point in the solution produced by the method offers a different perspective to the shock wave phenomenon. If the desired derivatives of the Eq. (5) are calculated and substituting in the Eq. (4), a polynomial with the argument $\left(1 / G^{\prime}\right)$ is attained. An algebraic equation is created by equalizing the coefficients of this polynomial to zero. These equations are solved with the help of a package program and put into place in the default Eq. (4) solution function. Finally, the solutions of Eq. (1) and Eq. (2) are obtained.

\section{Application of $\left(1 / G^{\prime}\right)$-expansion method}

\subsection{The solution of Tzitzéica equation}

We consider Eq. (1) by employing transformation $u=\ln v$, we get

$$
v v_{t t}-v_{t}^{2}-v v_{x x}+v_{x}^{2}-v^{3}+1=0 .
$$

We want to get the different type of exact solutions for the Eq. (8) by using $\left(1 / G^{\prime}\right)$-expansion method, with the help of transmutation $u=\ln v$, the general solution of Eq. (8) can be found. Suppose that the solution Eq. (8) is in the following

$$
v=V(\xi), \quad \xi=k x+w t,
$$

where $k, w$ are real numbers. Replacing Eq. (9) into Eq. (8) for obtaining nonlinear ODE:

$$
\left(w^{2}-k^{2}\right) V V^{\prime \prime}+\left(k^{2}-w^{2}\right)\left(V^{\prime}\right)^{2}-V^{3}+1=0 .
$$

In Eq. (10), we find balancing term say $N=2$ and in Eq. (5), solving both the following situation is obtained

$$
V=a_{0}+a_{1}\left(\frac{1}{G^{\prime}}\right)+a_{2}\left(\frac{1}{G^{\prime}}\right)^{2}, \quad a_{1} \neq 0 \quad \text { or } \quad a_{2} \neq 0 .
$$

Replacing Eq. (11) into Eq. (10) and the coefficients of the algebraic Eq. (1) are equal to zero, we can establish the following algebraic equation systems:

$$
\left(\frac{1}{G^{\prime}[\xi]}\right)^{0}: 1-a_{0}^{3}=0
$$

$$
\left(\frac{1}{G^{\prime}[\xi]}\right)^{1}:-k^{2} \lambda^{2} a_{0} a_{1}+w^{2} \lambda^{2} a_{0} a_{1}-3 a_{0}^{2} a_{1}=0,
$$




$$
\begin{gathered}
\left(\frac{1}{G^{\prime}[\xi]}\right)^{2}:-3 k^{2} \lambda \mu a_{0} a_{1}+3 w^{2} \lambda \mu a_{0} a_{1}-3 a_{0} a_{1}^{2}-4 k^{2} \lambda^{2} a_{0} a_{2}+4 w^{2} \lambda^{2} a_{0} a_{2}-3 a_{0}^{2} a_{2}=0, \\
\left(\frac{1}{G^{\prime}[\xi]}\right)^{3}:-2 k^{2} \mu^{2} a_{0} a_{1}+2 w^{2} \mu^{2} a_{0} a_{1}-k^{2} \lambda \mu a_{1}^{2}+w^{2} \lambda \mu a_{1}^{2}-a_{1}^{3}-10 k^{2} \lambda \mu a_{0} a_{2} \\
+10 w^{2} \lambda \mu a_{0} a_{2}-k^{2} \lambda^{2} a_{1} a_{2}+w^{2} \lambda^{2} a_{1} a_{2}-6 a_{0} a_{1} a_{2}=0, \\
\left(\frac{1}{G^{\prime}[\xi]}\right)^{4}:-k^{2} \mu^{2} a_{1}^{2}+w^{2} \mu^{2} a_{1}^{2}-6 k^{2} \mu^{2} a_{0} a_{2}+6 w^{2} \mu^{2} a_{0} a_{2}-5 k^{2} \lambda \mu a_{1} a_{2}+5 w^{2} \lambda \mu a_{1} a_{2}-3 a_{1}^{2} a_{2}-3 a_{0} a_{2}^{2}=0, \\
\left(\frac{1}{G^{\prime}[\xi]}\right)^{5}:-4 k^{2} \mu^{2} a_{1} a_{2}+4 w^{2} \mu^{2} a_{1} a_{2}-2 k^{2} \lambda \mu a_{2}^{2}+2 w^{2} \lambda \mu a_{2}^{2}-3 a_{1} a_{2}^{2}=0, \\
\left(\frac{1}{G^{\prime}[\xi]}\right)^{6}:-2 k^{2} \mu^{2} a_{2}^{2}+2 w^{2} \mu^{2} a_{2}^{2}-a_{2}^{3}=0 .
\end{gathered}
$$

Case1.

$$
a_{0}=1, \quad a_{1}=\frac{6 \mu}{\lambda}, \quad a_{2}=\frac{6 \mu^{2}}{\lambda^{2}}, \quad k=-\frac{\sqrt{-3+w^{2} \lambda^{2}}}{\lambda}, \quad \xi=k x+w t .
$$

Considering Eq. (7) and substituting in Eq. (13) and Eq. (11), the following solution is obtained.

$$
\begin{aligned}
& V=1+\frac{6 \mu^{2}}{\lambda^{2}\left(-\frac{\mu}{\lambda}+A \cosh \left[\lambda\left(t w-\frac{x \sqrt{-3+w^{2} \lambda^{2}}}{\lambda}\right)\right]-A \sinh \left[\lambda\left(t w-\frac{x \sqrt{-3+w^{2} \lambda^{2}}}{\lambda}\right)\right]\right)^{2}} \\
& +\frac{6 \mu}{\lambda\left(-\frac{\mu}{\lambda}+A \cosh \left[\lambda\left(t w-\frac{x \sqrt{-3+w^{2} \lambda^{2}}}{\lambda}\right)\right]-A \sinh \left[\lambda\left(t w-\frac{x \sqrt{-3+w^{2} \lambda^{2}}}{\lambda}\right)\right]\right)},
\end{aligned}
$$

In addition, if Eq. (14) is written instead of $u=\ln v$ transformation, the analytical solution of Eq. (1) is as follows.

$$
\begin{aligned}
u_{1}(x, t)= & \ln \left[1+\frac{6 \mu^{2}}{\lambda^{2}\left(-\frac{\mu}{\lambda}+A \cosh \left[\lambda\left(t w-\frac{x \sqrt{-3+w^{2} \lambda^{2}}}{\lambda}\right)\right]-A \sinh \left[\lambda\left(t w-\frac{x \sqrt{-3+w^{2} \lambda^{2}}}{\lambda}\right)\right]\right)^{2}}\right. \\
& \left.+\frac{6 \mu}{\lambda\left(-\frac{\mu}{\lambda}+A \cosh \left[\lambda\left(t w-\frac{x \sqrt{-3+w^{2} \lambda^{2}}}{\lambda}\right)\right]-A \sinh \left[\lambda\left(t w-\frac{x \sqrt{-3+w^{2} \lambda^{2}}}{\lambda}\right)\right]\right)}\right] .
\end{aligned}
$$

\section{Case 2.}

$$
a_{0}=\frac{1}{2}(-1+\mathrm{i} \sqrt{3}), \quad a_{1}=\frac{3 \mathrm{i}(\mathrm{i} \mu+\sqrt{3} \mu)}{\lambda}, \quad a_{2}=\frac{3 \mathrm{i}\left(\mathrm{i} \mu^{2}+\sqrt{3} \mu^{2}\right)}{\lambda^{2}}, \quad k=-\sqrt{w^{2}+\frac{3}{2 \lambda^{2}}-\frac{3 \mathrm{i} \sqrt{3}}{2 \lambda^{2}}}, \quad \xi=k x+w t,
$$

where $\mathrm{i}=\sqrt{-1}$. Considering Eq. (7), substituting Eq. (16) into Eq. (11), the following solution is obtained.

$$
\begin{aligned}
V=\frac{1}{2}( & -1+\mathrm{i} \sqrt{3})+\frac{3 \mathrm{i}\left(\mathrm{i} \mu^{2}+\sqrt{3} \mu^{2}\right)}{\lambda^{2}\left(-\frac{\mu}{\lambda}+A \cosh \left[\left(t w+x \sqrt{w^{2}+\frac{3}{2 \lambda^{2}}-\frac{3 \mathrm{i} \sqrt{3}}{2 \lambda^{2}}}\right) \lambda\right]-A \sinh \left[\left(t w+x \sqrt{w^{2}+\frac{3}{2 \lambda^{2}}-\frac{3 \mathrm{i} \sqrt{3}}{2 \lambda^{2}}}\right) \lambda\right]\right)^{2}} \\
+ & \frac{3 \mathrm{i}(\mathrm{i} \mu+\sqrt{3} \mu)}{\lambda\left(-\frac{\mu}{\lambda}+A \cosh \left[\left(t w+x \sqrt{w^{2}+\frac{3}{2 \lambda^{2}}-\frac{3 \mathrm{i} \sqrt{3}}{2 \lambda^{2}}}\right) \lambda\right]-A \sinh \left[\left(t w+x \sqrt{w^{2}+\frac{3}{2 \lambda^{2}}-\frac{3 \mathrm{i} \sqrt{3}}{2 \lambda^{2}}}\right) \lambda\right]\right)} \cdot
\end{aligned}
$$

In addition, if Eq. (17) is written instead of $u=\ln v$ transformation, the analytical solution of Eq. (1) is as follows.

$$
\begin{aligned}
u_{2}(x, t)= & \ln \left[\frac{1}{2}(-1+\mathrm{i} \sqrt{3})+\frac{3 \mathrm{i}\left(\mathrm{i} \mu^{2}+\sqrt{3} \mu^{2}\right)}{\lambda^{2}\left(-\frac{\mu}{\lambda}+A \cosh \left[\left(t w+x \sqrt{w^{2}+\frac{3}{2 \lambda^{2}}-\frac{3 \mathrm{i} \sqrt{3}}{2 \lambda^{2}}}\right) \lambda\right]-A \sinh \left[\left(t w+x \sqrt{w^{2}+\frac{3}{2 \lambda^{2}}-\frac{3 \mathrm{i} \sqrt{3}}{2 \lambda^{2}}}\right) \lambda\right]\right)^{2}}\right. \\
& \left.+\frac{3 \mathrm{i}(\mathrm{i} \mu+\sqrt{3} \mu)}{\lambda\left(-\frac{\mu}{\lambda}+A \cosh \left[\left(t w+x \sqrt{w^{2}+\frac{3}{2 \lambda^{2}}-\frac{3 \mathrm{i} \sqrt{3}}{2 \lambda^{2}}}\right) \lambda\right]-A \sinh \left[\left(t w+x \sqrt{w^{2}+\frac{3}{2 \lambda^{2}}-\frac{3 \mathrm{i} \sqrt{3}}{2 \lambda^{2}}}\right) \lambda\right]\right)}\right] \cdot(18)
\end{aligned}
$$




\section{Case 3.}

$$
\begin{aligned}
& a_{0}=1, \quad a_{1}=2 \sqrt{3} \sqrt{-k^{2} \mu^{2}+w^{2} \mu^{2}}, \quad a_{2}=2\left(-k^{2} \mu^{2}+w^{2} \mu^{2}\right), \\
& \lambda=\left(\frac{\sqrt{3} \sqrt{\left[\left(-k^{2}+w^{2}\right) \mu^{2}\right]}}{\left(-k^{2} \mu+w^{2} \mu\right)}\right), \quad \xi=k x+w t .
\end{aligned}
$$

Considering Eq. (7), substituting Eq. (19) into Eq. (11), the following solution is obtained.

$$
V=\frac{9 A^{2}+3 \mathrm{e}^{\frac{2 \sqrt{3}(t w+k x) \mu}{\sqrt{\left(-k^{2}+w^{2}\right) \mu^{2}}}}(-k+w)(k+w) \mu^{2}+12 \sqrt{3} A \mathrm{e}^{\frac{\sqrt{3}(t w+k x) \mu}{\sqrt{\left(-k^{2}+w^{2}\right) \mu^{2}}}} \sqrt{\left(-k^{2}+w^{2}\right) \mu^{2}}}{\left(-3 A+\sqrt{3} e^{\frac{\sqrt{3}(t w+k x) \mu}{\sqrt{\left(-k^{2}+w^{2}\right) \mu^{2}}}} \sqrt{\left(-k^{2}+w^{2}\right) \mu^{2}}\right)^{2}} .
$$

In addition, if Eq. (20) is written instead of $u=\ln v$ transformation, the analytical solution of Eq. (1) is as follows.

$$
u_{3}(x, t)=\ln \left[\frac{9 A^{2}+3 \mathrm{e}^{\frac{2 \sqrt{3}(t w+k x) \mu}{\sqrt{\left(-k^{2}+w^{2}\right) \mu^{2}}}}(-k+w)(k+w) \mu^{2}+12 \sqrt{3} A \mathrm{e}^{\frac{\sqrt{3}(t w+k x) \mu}{\sqrt{\left(-k^{2}+w^{2}\right) \mu^{2}}}} \sqrt{\left(-k^{2}+w^{2}\right) \mu^{2}}}{\left(-3 A+\sqrt{3} \mathrm{e}^{\frac{\sqrt{3}(t w+k x) \mu}{\sqrt{\left(-k^{2}+w^{2}\right) \mu^{2}}}} \sqrt{\left(-k^{2}+w^{2}\right) \mu^{2}}\right)^{2}}\right] .
$$

\subsection{The solution of the Dodd-Bullough-Mikhailov equation}

We consider Eq. (2). Using transmutation $u=\ln v$, we get

$$
v v_{x t}-v_{x} v_{t}+v^{3}+1=0
$$

Suppose that solution of Eq. (22) may be write as follows

$$
v=V(\xi), \quad \xi=k x+w t
$$

where $k, w$ are real numbers. Replacing Eq. (23) into Eq. (22) to obtain nonlinear ODE:

$$
k w V V^{\prime \prime}-k w\left(V^{\prime}\right)^{2}+V^{3}+1=0 .
$$

In Eq. (24), we obtain balancing term $N=2$, then the following relationship is achieved:

$$
V=a_{0}+a_{1}\left(\frac{1}{G^{\prime}}\right)+a_{2}\left(\frac{1}{G^{\prime}}\right)^{2}, \quad a_{1} \neq 0 \quad \text { or } \quad a_{2} \neq 0
$$

Replacing Eq. (25) into Eq. (24), and the coefficients of the algebraic of Eq. (2) are equal to zero, we may find the following algebraic equation systems,

$$
\begin{gathered}
\left(\frac{1}{G^{\prime}[\xi]}\right)^{0}: 1+a_{0}^{3}=0, \\
\left(\frac{1}{G^{\prime}[\xi]}\right)^{1}: k w \lambda^{2} a_{0} a_{1}+3 a_{0}^{2} a_{1}=0, \\
\left(\frac{1}{G^{\prime}[\xi]}\right)^{2}: 3 k w \lambda \mu a_{0} a_{1}+3 a_{0} a_{1}^{2}+4 k w \lambda^{2} a_{0} a_{2}+3 a_{0}^{2} a_{2}=0, \\
\left(\frac{1}{G^{\prime}[\xi]}\right)^{3}: 2 k w \mu^{2} a_{0} a_{1}+k w \lambda \mu a_{1}^{2}+a_{1}^{3}+10 k w \lambda \mu a_{0} a_{2}+k w \lambda^{2} a_{1} a_{2}+6 a_{0} a_{1} a_{2}=0, \\
\left(\frac{1}{G^{\prime}[\xi]}\right)^{4}: k w \mu^{2} a_{1}^{2}+6 k w \mu^{2} a_{0} a_{2}+5 k w \lambda \mu a_{1} a_{2}+3 a_{1}^{2} a_{2}+3 a_{0} a_{2}^{2}=0, \\
\left(\frac{1}{G^{\prime}[\xi]}\right)^{5}: 4 k w \mu^{2} a_{1} a_{2}+2 k w \lambda \mu a_{2}^{2}+3 a_{1} a_{2}^{2}=0,
\end{gathered}
$$




$$
\left(\frac{1}{G^{\prime}[\xi]}\right)^{6}: 2 k w \mu^{2} a_{2}^{2}+a_{2}^{3}=0
$$

Case 1.

$$
a_{0}=-1, \quad a_{1}=2 \sqrt{3} \sqrt{k} \sqrt{w} \mu, \quad a_{2}=-2 k w \mu^{2}, \quad \lambda=-\frac{\sqrt{3}}{\sqrt{k} \sqrt{w}}, \quad \xi=k x+w t .
$$

Considering Eq. (7), substituting Eq. (27) into Eq. (25), the following solution is obtained.

$$
V=-1-\frac{2 k w \mu^{2}}{\left(\frac{\sqrt{k} \sqrt{w} \mu}{\sqrt{3}}+A \cosh \left[\frac{\sqrt{3}(t w+k x)}{\sqrt{k} \sqrt{w}}\right]+A \sinh \left[\frac{\sqrt{3}(t w+k x)}{\sqrt{k} \sqrt{w}}\right]\right)^{2}}+\frac{2 \sqrt{3} \sqrt{k} \sqrt{w} \mu}{\frac{\sqrt{k} \sqrt{w} \mu}{\sqrt{3}}+A \cosh \left[\frac{\sqrt{3}(t w+k x)}{\sqrt{k} \sqrt{w}}\right]+A \sinh \left[\frac{\sqrt{3}(t w+k x)}{\sqrt{k} \sqrt{w}}\right]} .
$$

In addition, if Eq. (28) is written instead of $u=\ln v$ transformation, the analytical solution of Eq. (2) is as follows.

$u_{1}(x, t)=\ln \left[-1-\frac{2 k w \mu^{2}}{\left(\frac{\sqrt{k} \sqrt{w} \mu}{\sqrt{3}}+A \cosh \left[\frac{\sqrt{3}(t w+k x)}{\sqrt{k} \sqrt{w}}\right]+A \sinh \left[\frac{\sqrt{3}(t w+k x)}{\sqrt{k} \sqrt{w}}\right]\right)^{2}}+\frac{2 \sqrt{3} \sqrt{k} \sqrt{w} \mu}{\frac{\sqrt{k} \sqrt{w} \mu}{\sqrt{3}}+A \cosh \left[\frac{\sqrt{3}(t w+k x)}{\sqrt{k} \sqrt{w}}\right]+A \sinh \left[\frac{\sqrt{3}(t w+k x)}{\sqrt{k} \sqrt{w}}\right]}\right]$.

\section{Case 2.}

$$
a_{0}=\frac{1}{2}(1+\mathrm{i} \sqrt{3}), a_{1}=-\sqrt{6} \sqrt{-k w \mu^{2}-\mathrm{i} \sqrt{3} k w \mu^{2}}, a_{2}=-2 k w \mu^{2}, \lambda=\frac{\sqrt{\frac{3}{2}} \sqrt{-\mathrm{i}(-\mathrm{i}+\sqrt{3}) k w \mu^{2}}}{k w \mu}, \xi=k x+w t .
$$

Considering Eq. (7), substituting Eq. (30) into Eq. (25), the following solution is obtained

$$
\begin{aligned}
& V=\frac{1}{2}(1+\mathrm{i} \sqrt{3})
\end{aligned}
$$

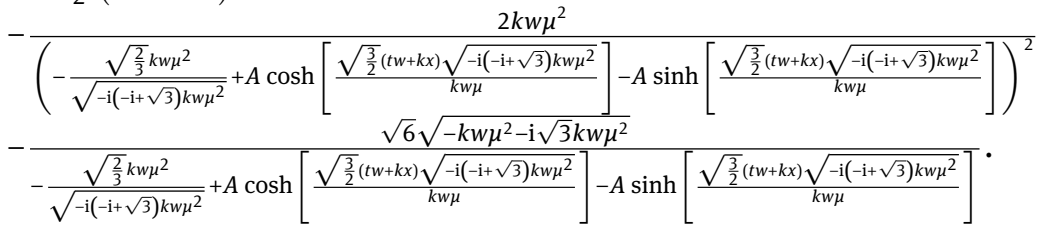

In addition, if Eq. (31) is written instead of $u=\ln v$ transformation, the analytical solution of Eq. (2) is as follows

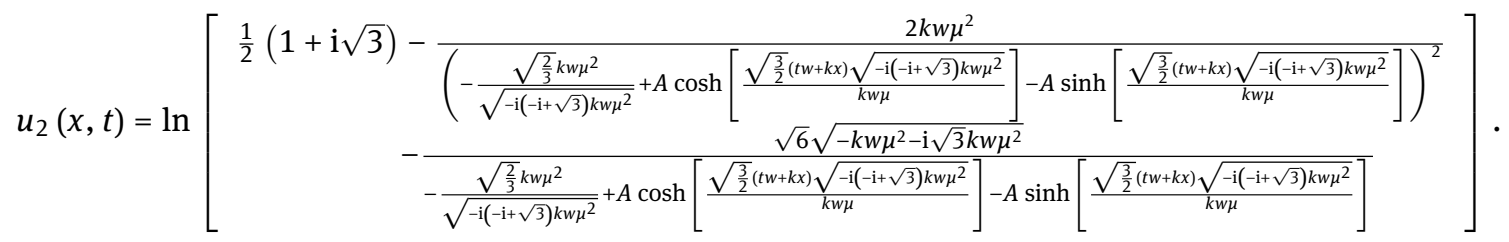
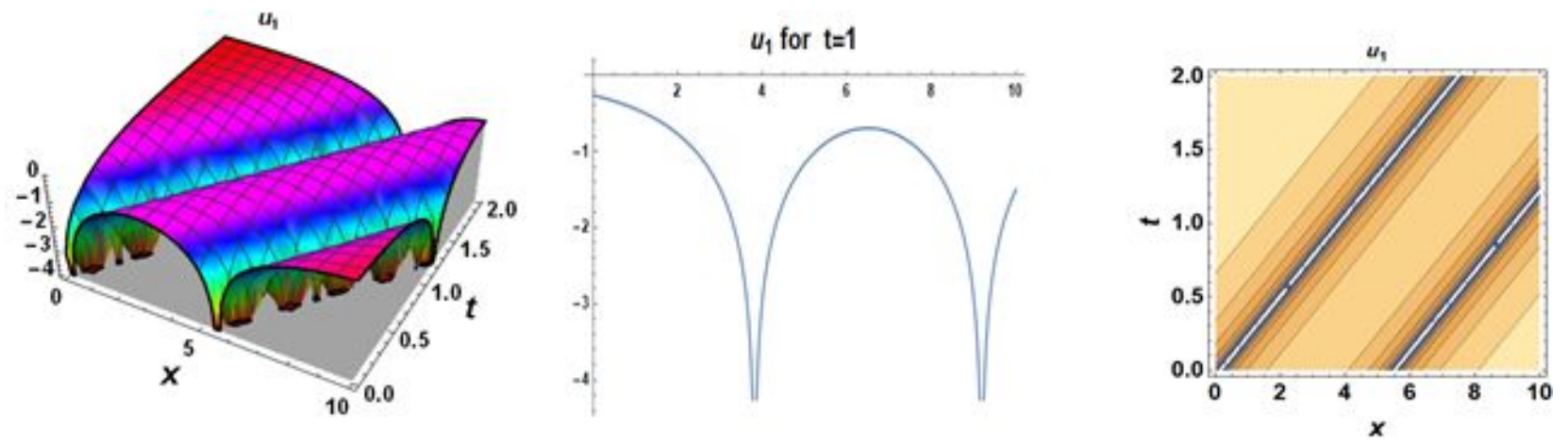

Figure 1: 3D,2D and contour graphics for $A=1, \mu=-4, \lambda=1, w=1.8$ values of Eq. (1). 


\section{Results and discussion}

One of the important instruments used in obtaining the exact solution of nonlinear evolution equations is the expansion method. In the literature, traveling wave solutions have been obtained by using many expansion methods. The different types of traveling wave solutions were constructed from $\left(G^{\prime} / G\right)$-expansion method, novel exponential rational function method, sub equation method, extended auxiliary equation method, the tanh method, Kudryashov method, Sine-Gordon expansion method, the extended tanh method, the improved $\tan (\phi(\xi) / 2)$ expansion method, auxiliary equation method and the extended trial equation method and several others. The analytic solution function is obtained by employed method for Eq. (7). In addition, the solution presented with the Eq. (21) contains the exponential solution for the basic equation. Such solution is similar to the format of Eq. (7) but the coefficients are different when the solution in Eq. (21) is written in trigonometric form. The Tzitzéica and the Dodd-Bullough-Mikhailov equations are same family as presented by using two transformations with $\left(1 / G^{\prime}\right)$-expansion method. Different arguments can be created by giving special values to constants in the solutions. The Tzitzéica and the Dodd-Bullough-Mikhailov equations contain an exponential function that indicates the solution function in logarithmic form. On the basis of $u=\ln v$ transformation, it verifies that the solutions are logarithmic in which $v$ is the hyperbolic traveling wave solution. In addition, mobility of the wave can be achieved by giving appropriate values to the constants among the solutions. Let us specifically analyze the traveling wave solution presented by Eq. (15).

$$
\lim _{x \rightarrow \pm \infty} u_{1}=0
$$

Considering the Eq. (33), it can be said that Eq. (15) exhibits a damped wave behavior independent of the variable $t$. Because for $x \rightarrow \pm \infty$ is $u_{1} \rightarrow 0$. In addition, we can observe the progress of the following solitary wave for different values of $t$ time parameter, $x \in[0,25]$ of the traveling wave solution presented by Eq. (15). Figure 6 presents that a wave moves to the right. One of the most important reasons for this is that $w>0$ represents the velocity of the wave. $w=1.8$ that is chosen for Figure 6 .

\section{Conclusions}

In this article, we have obtained traveling wave solutions for the Tzitzéica and the Dodd-Bullough-Mikhailov equations by invoking of $\left(1 / G^{\prime}\right)$-expansion method. For both equations, the solutions are obtained in hyperbolic form. The validity of the $\left(1 / G^{\prime}\right)$-expansion method has been tested by checking the hyperbolic type solutions. Consequently, nonlinear part of the method can be recommended for generating differential equations in traveling wave solutions. The 3D, 2D and contour graphics representing the constant waves are presented by evaluating the constants in the traveling wave solutions. This method is an important instrument in obtaining the traveling wave solutions of nonlinear evolution equations. The reliability of this method can be used to obtain the analytical solution of differential equations in different classes. Due to this fact, this method increases the importance of its methodology. To conclude that complex processes and difficulties have been overcome with the help of computer programs.

Acknowledgements: The author Dr. Hülya Durur is highly thankful and grateful to Ardahan University, Ardahan, Turkey for generous support and facilities of this research work. The author Dr. Asıf Yokuş is highly thankful and grateful to Firat University, Elazig, Turkey for generous support and facilities of this research work. The author Dr. Kashif Ali Abro is highly thankful and grateful to Mehran university of Engineering and Technology, Jamshoro, Pakistan for generous support and facilities of this research work.

Funding information: The authors state no funding involved.

Author contributions: All authors have accepted responsibility for the entire content of this manuscript and approved its submission.

Conflict of interest: The authors state no conflict of interest.

Data Availability Statement: The datasets generated during and/or analysed during the current study are available from the corresponding author on reasonable request. 

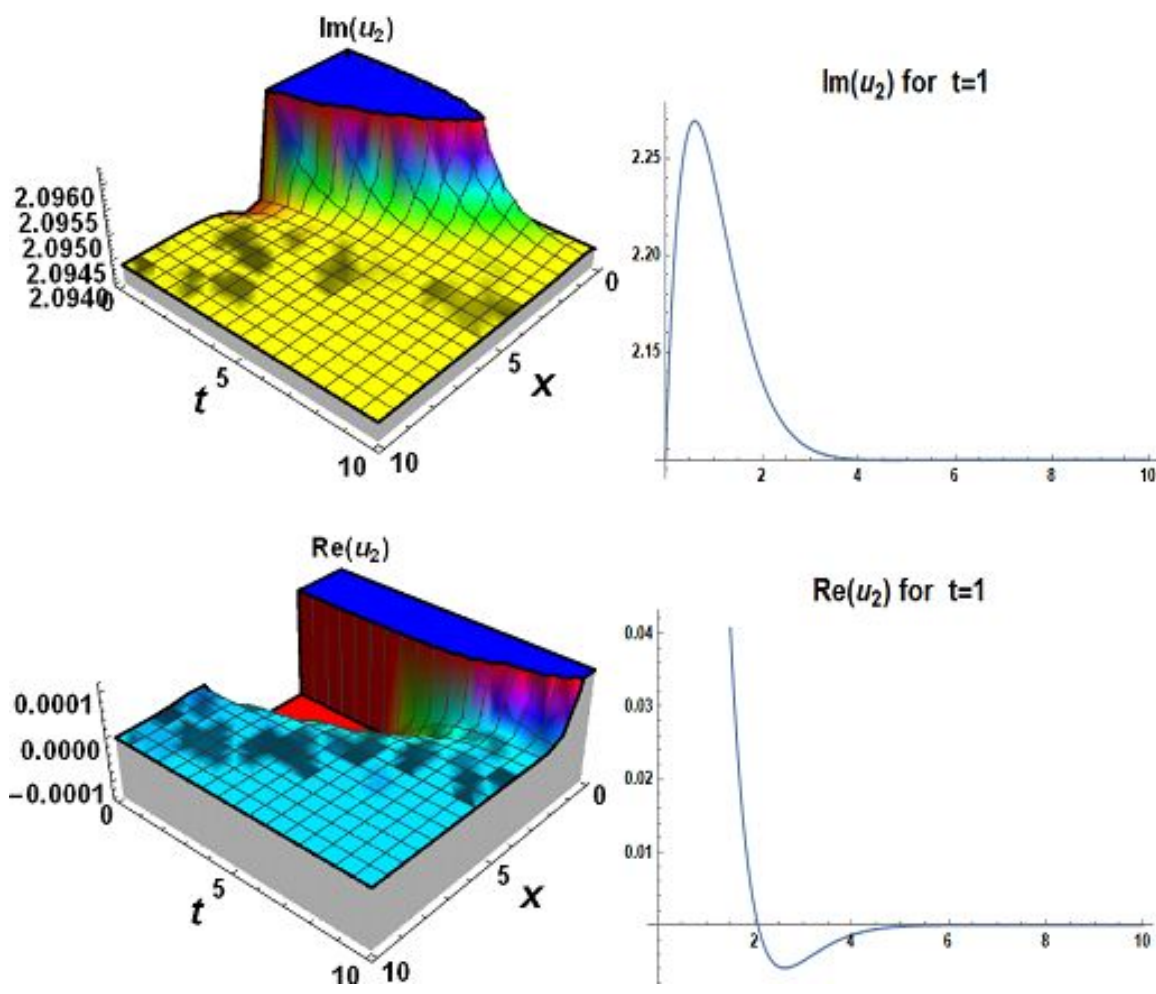

Figure 2: 3D,2D and contour graphics for $A=-2, \mu=-2, \lambda=0.5, w=1.8$ values of Eq. (1).
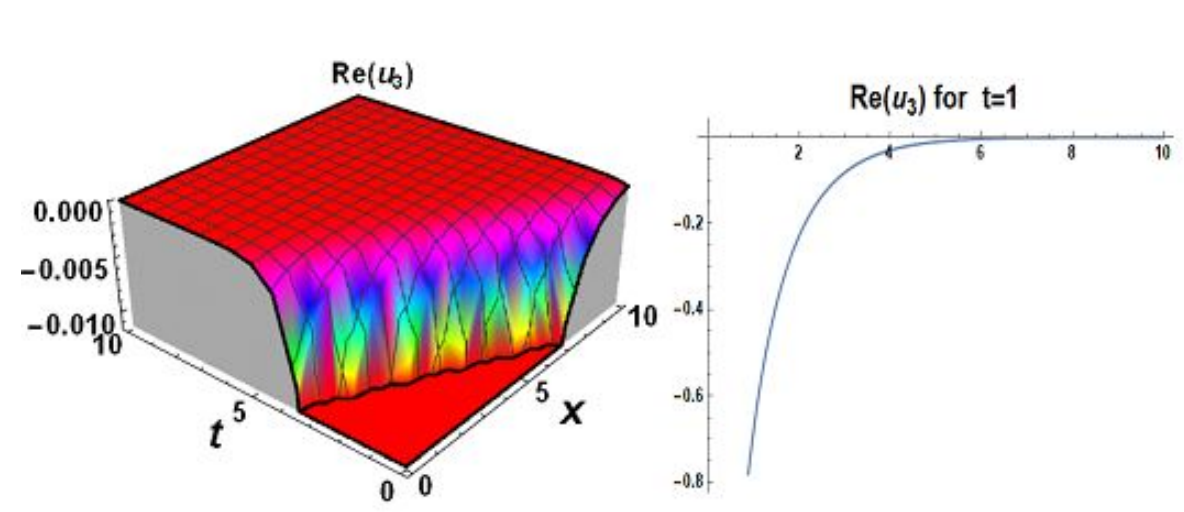

Figure 3: 3D,2D and contour graphics for $A=-2, \mu=-4, \lambda=2, w=2, k=1$ values of Eq. (1).


Figure 4: 3D,2D and contour graphics for $A=1, \mu=-1, \lambda=-1, w=1, k=2$ values of Eq. (2). 

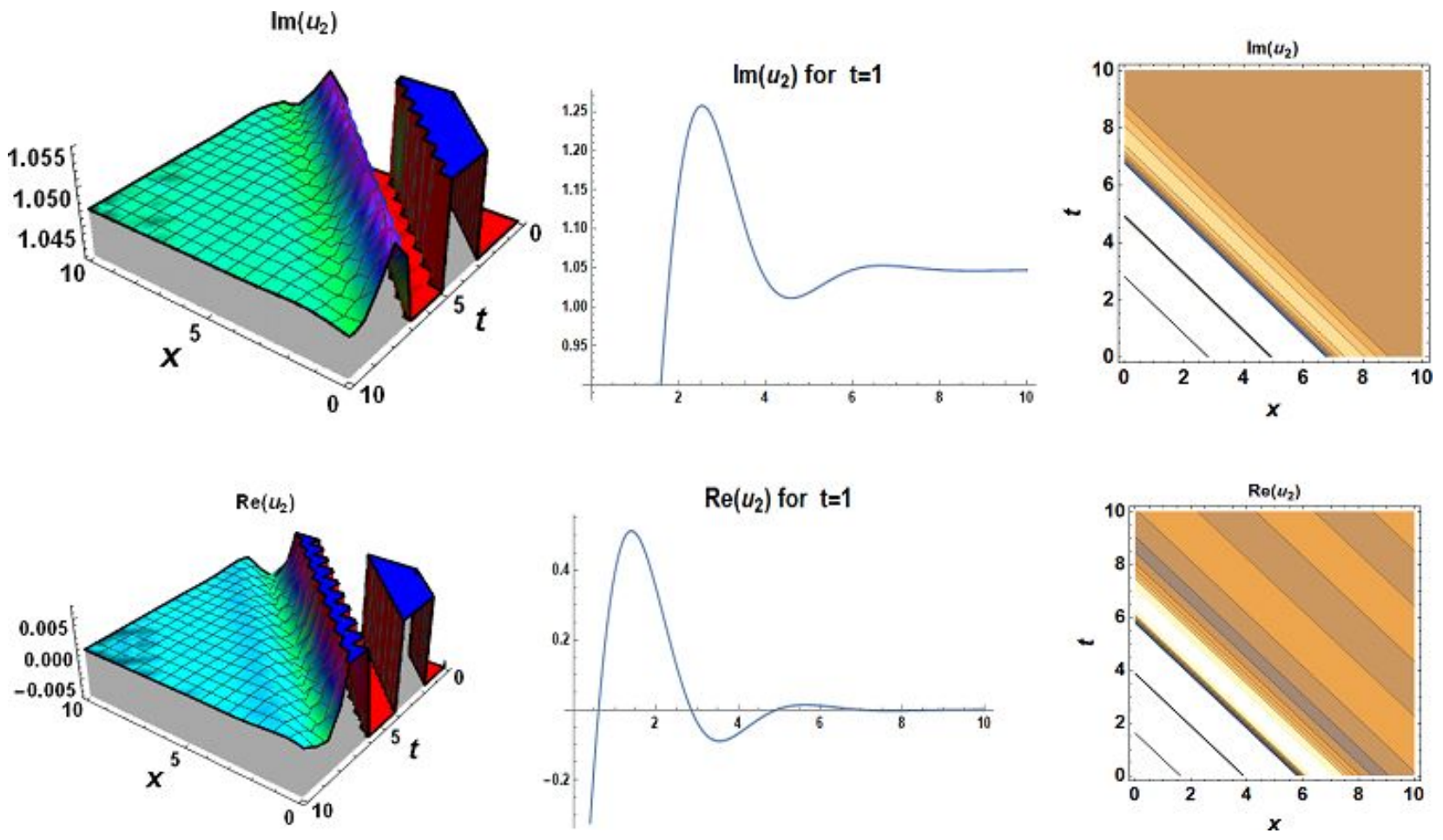

Figure 5: 3D,2D and contour graphics for $A=1, \mu=2, \lambda=1, w=1, k=1$ values of Eq. (2).
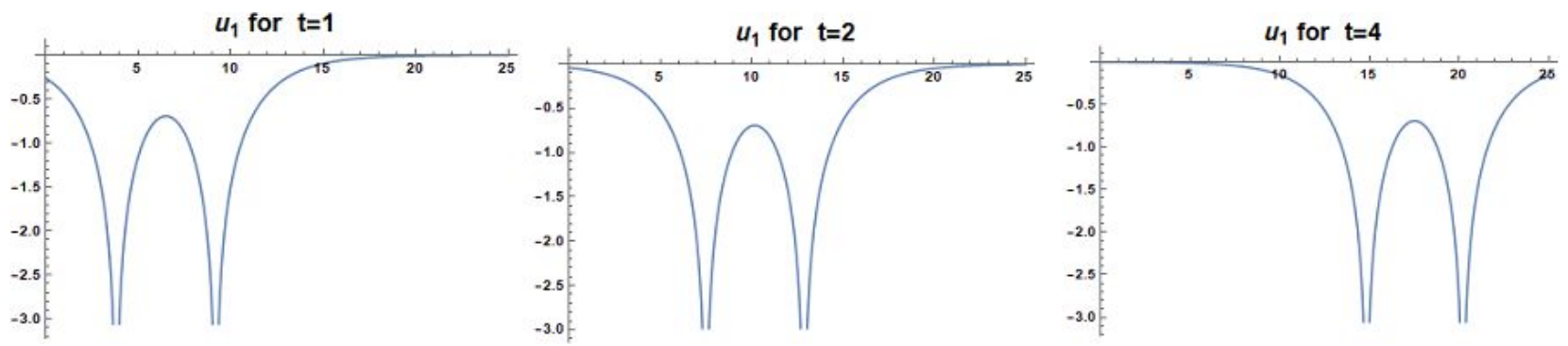

Figure 6: Simulation graphics for $A=1, \mu=-4, \lambda=1, w=1.8$ values of Eq. (15).

\section{References}

[1] Baskonus HM, Sulaiman TA, Bulut H, Aktürk T. Investigations of dark, bright, combined dark-bright optical and other soliton solutions in the complex cubic nonlinear Schrödinger equation with $\delta$-potential. Superlattices Microstruct. 2018;115:1929.

[2] Cattani C, Sulaiman TA, Baskonus HM, Bulut H. On the soliton solutions to the Nizhnik-Novikov-Veselov and the Drinfel'dSokolov systems. Opt Quantum Electron. 2018;50(3):138.

[3] Bulut H, Yel G, Başkonuş HM. An application of improved Bernoulli sub-equation function method to the nonlinear timefractional burgers equation. Turkish Journal of Mathematics and Computer Science. 2016;5:1-7.

[4] Dusunceli F, Celik E, Askin M. New exact solutions for the doubly dispersive equation using an improved Bernoulli subequation function method. Indian J Phys. 2021;95(3):309-14.
[5] Miah MM, Ali HS, Akbar MA, Seadawy AR. New applications of the two variable $(G / / G, 1 / G)$-expansion method for closed form traveling wave solutions of integro-differential equations. J Ocean Eng Sci. 2019;4(2):132-43.

[6] Yokuş A, Kaya D. Traveling wave solutions of some nonlinear partial differential equations by using extended-expansion method. 2015;54(2):263-69.

[7] Yokus A, Tuz M. An application of a new version of (G//G)expansion method. No. 1. AIP Conference Proceedings. Volume 1798. AIP Publishing; 2017:020165.

[8] Durur H. Different types analytic solutions of the (1+ 1$)$ dimensional resonant nonlinear Schrödinger's equation using (G//G)-expansion method. Mod Phys Lett $B$. 2020;34(3):2050036.

[9] Durur H, Taşbozan O, Kurt A, Şenol M. New Wave Solutions of Time Fractional Kadomtsev-Petviashvili Equation Arising In the Evolution of Nonlinear Long Waves of Small Amplitude. Erzincan Univ J Inst Sci Technol. 2019;12(2):807-15. 
[10] Yokuş A, Durur H. Complex hyperbolic traveling wave solutions of Kuramoto-Sivashinsky equation using (1/G/) expansion method for nonlinear dynamic theory. Journal of Balıkesir University Institute of Science and Technology. 2019;21(2):590-9.

[11] Yokuş A, Kaya D. Conservation laws and a new expansion method for sixth order Boussinesq equation. No. 1. AIP Conference Proceedings. Volume 1676. AIP Publishing; 2015:020062.

[12] Durur H, Yokuş A. Hyperbolic Traveling Wave Solutions for Sawada-Kotera Equation Using -Expansion Method. Afyon Kocatepe University Journal of Science and Engineering. 2019;19(3):615-9.

[13] Durur H, Şenol M, Kurt A, Taşbozan O. Zaman-Kesirli Kadomtsev-Petviashvili Denkleminin Conformable Türev ile Yaklaşık Çözümleri. Erzincan Univ J Inst Sci Technol. 2019;12(2):796-806.

[14] Kaya D, Yokus A. A numerical comparison of partial solutions in the decomposition method for linear and nonlinear partial differential equations. Math Comput Simul. 2002;60(6):50712.

[15] Kaya D, Yokus A. A decomposition method for finding solitary and periodic solutions for a coupled higher-dimensional Burgers equations. Appl Math Comput. 2005;164(3):857-64.

[16] Yokus A, Kaya D. A numerical comparison for coupled boussinesq equations by using the ADM. Proc. 2004:730-36.

[17] Yavuz M, Özdemir N. A quantitative approach to fractional option pricing problems with decomposition series. Konuralp Journal of Mathematics. 2018;6(1):102-9.

[18] Yavuz M, Özdemır N. An Integral Transform Solution for Fractional Advection-Diffusion Problem. Math Stud Appl. 2018;46:442.

[19] Šarler B, Aziz I. Haar wavelet collocation method for the numerical solution of boundary layer fluid flow problems. Int J Therm Sci. 2011;50(5):686-97.

[20] Aziz I, Asif M. Haar wavelet collocation method for threedimensional elliptic partial differential equations. Comput Math Appl. 2017;73(9):2023-34.

[21] Darvishi M, Arbabi S, Najafi M, Wazwaz A. Traveling wave solutions of a (2+1)-dimensional Zakharov-like equation by the first integral method and the tanh method. Optik (Stuttg). 2016;127(16):6312-21.

[22] Abazari R. The (G/G)-expansion method for Tzitzéica type nonlinear evolution equations. Math Comput Model. 2010;52(910):1834-45.

[23] Kumar D, Hosseini K, Samadani F. The sine-Gordon expansion method to look for the traveling wave solutions of the Tzitzéica type equations in nonlinear optics. Optik (Stuttg). 2017;149:439-46.

[24] Huber A. A note on a class of solitary-like solutions of the Tzitzéica equation generated by a similarity reduction. Physica D. $2008 ; 237(8): 1079-87$.

[25] Wazwaz AM. The tan $\mathrm{h}$ method: solitons and periodic solutions for the Dodd-Bullough-Mikhailov and the Tzitzeica-DoddBullough equations. Chaos Solitons Fractals. 2005;25(1):5563.

[26] Manafian J, Lakestani M. Dispersive dark optical soliton with Tzitzéica type nonlinear evolution equations arising in nonlinear optics. Opt Quantum Electron. 2016;48(2):116.

[27] Hosseini K, Ayati Z, Ansari R. New exact traveling wave solutions of the Tzitzéica type equations using a novel exponential rational function method. Optik (Stuttg). 2017;148:85-9.

[28] Hosseini K, Ayati Z, Ansari R. New exact solutions of the Tzitzéica-type equations in non-linear optics using the exp a function method. J Mod Opt. 2018;65(7):847-51.

[29] Babalic C, Constantinescu R, Gerdjikov VS. Two soliton solution of Tzitzeica equation. Physics AUC. 2013;23:36-41.

[30] Kashif AA, Abdon A. Porous effects on the fractional modeling of magnetohydrodynamic pulsatile flow: an analytic study via strong kernels. J Therm Anal Calorim. 2020; https://doi.org/10.1007/s10973-020-10027-z.

[31] Hamid M, Usman M, Zubair T, Haq RU, Shafee A. An efficient analysis for $\mathrm{N}$-soliton, Lump and lump-kink solutions of timefractional (2+1)-Kadomtsev-Petviashvili equation. Physica A. 2019;528:121320.

[32] Kashif AA, Atangana A. A comparative analysis of electromechanical model of piezoelectric actuator through CaputoFabrizio and Atangana-Baleanu fractional derivatives. Math Methods Appl Sci. 2020;43(17):1-11.

[33] Abro KA, Jose FG. Role of Fourier sine transform on the dynamical model of tensioned carbon nanotubes with fractional operator. Math Methods Appl Sci. 2020:1-11.

[34] Hamid M, Usman M, Wang W, Tian Z. Hybrid fully spectral linearized scheme for time-fractional evolutionary equations. Math Methods Appl Sci. 2021;44(5):3890-912.

[35] Aziz UA, Mukarram A, Kashif AA. Electroosmotic slip flow of Oldroyd-B fluid between two plates with nonsingular kernel. J Comput Appl Math. 2020;376:112885-99. https://doi.org/10.1016/j.cam.2020.112885.

[36] Bhojraj L, Kashif AA, Abdul WS. Thermodynamical analysis of heat transfer of gravity-driven fluid flow via fractional treatment: an analytical study. J Therm Anal Calorim. 2020; https://doi.org/10.1007/s10973-020-09429-w.

[37] Kashif AA, Abdon A. A comparative study of convective fluid motion in rotating cavity via Atangana-Baleanu and CaputoFabrizio fractal-fractional differentiations. Eur Phys J Plus. 2020;135(2):226-42.

[38] Abro KA, Ambreen S, Abdon A. Thermal stratification of rotational second-grade fluid through fractional differential operators. J Therm Anal Calorim. 2020; https://doi.org/10.1007/s10973-020-09312-8.

[39] Hamid M, Usman M, Wang W, Tian Z. A stable computational approach to analyze semi-relativistic behavior of fractional evolutionary problems. Numer Methods Partial Differ Equ. 2020;num.22617.

[40] Hamid M, Usman M, Haq RU, Tian Z, Wang W. Linearized stable spectral method to analyze two-dimensional nonlinear evolutionary and reaction-diffusion models. Numer Methods Partial Differ Equ. 2020;num.22659.

[41] Duran S, Karabulut B. Nematicons in liquid crystals with Kerr Law by sub-equation method. Alex Eng J. 2021; https://doi.org/10.1016/j.aej.2021.06.077.

[42] Duran S. Extractions of travelling wave solutions of $(2+1)$ dimensional Boiti-Leon-Pempinelli system via $\left(\mathrm{G}^{\prime} / \mathrm{G}, 1 / \mathrm{G}\right)$ expansion method. Opt Quantum Electron. 2021;53(6):1-12.

[43] Duran S. Breaking theory of solitary waves for the Riemann wave equation in fluid dynamics. Int J Mod Phys B. 2021;35(9):2150130.

[44] Mehmet Y, Tukur AS, Abdullahi Y, Thabet A. The Schrodinger$\mathrm{KdV}$ equation of fractional order with Mittag-Leffler nonsingular kernel. Alex Eng J. 2021;60(2):2715-24. 
[45] Mehmet Y, Ndolane S. Approximate solutions of the model describing fluid flow using generalized-Laplace transform method and heat balance integral method. Axioms. 2020;9(4):123.

[46] Mehmet Y, Tukur AS, Fuat U, Hasan B. Analysis and numerical computations of the fractional regularized long-wave equation with damping term. Math Methods Appl Sci. 2021;44(9):753855.

[47] Mehmet Y, Necati O. Numerical Inverse Laplace Homotopy Technique for Fractional Heat Equations. Therm Sci. 2018;22(1 Suppl. 1):185-94. 